HNO 2011 · 59:744-745

DOI 10.1007/s00106-011-2348-2

Online publiziert: 3. Juni 2011

(c) Springer-Verlag 2011

\section{H. Maier}

Kopfklinik am Bundeswehrkrankenhaus Ulm, Klinik und Poliklinik für HNO-Heilkunde/ Kopf- und Halschirurgie, Akademisches Krankenhaus der Universität Ulm

\title{
Schuss- und Splitterverletzungen im Kopf- und Halsbereich
}

Verletzungen im Kopf- und Halsbereich sind in Deutschland nach wie vor überwiegend auf Verkehrs- und Sportunfälle zurückzuführen. Verletzungen durch Schusswaffen und/oder Sprengkörper sind im Vergleich zu anderen Ländern eher selten.

Betrachtet man die Tötungsdelikte mit Schusswaffen weltweit, so betrug die Häufigkeit in Deutschland nur o,19 Fälle pro 100.000 Einwohner. In den USA lag diese Zahl mit 3,45 Fällen um fast das 20-Fache und in Südafrika mit 30,17 Fällen um fast das 200-Fache höher. Für Kolumbien wurde sogar ein Wert von 49,54 Fällen pro 100.00o Einwohner mitgeteilt (Zeitspanne 1998 bis 2001; http://de.wikipedia.org/wiki/Waffenmissbrauch). Die Zahl der schussverletzten Personen dürfte vermutlich um ein Vielfaches höher liegen.

Diese Konstellation hat zur Folge, dass abgesehen von den Ärzten des Sanitätsdienstes der Bundeswehr, die eine spezielle Ausbildung besitzen und im Rahmen von Kampfeinsätzen ständig mit Schussund Explosionsverletzungen konfrontiert werden, die meisten Ärzte in Deutschland nur wenig Erfahrung mit der Versorgung von ballistischen Traumen besitzen. Tragische Ereignisse, wie z. B. der Amoklauf in Winnenden, aber auch die wachsende Bedrohung durch terroristische Anschläge in Europa, machen deutlich, dass auch zivile Ärzte mit der Diagnostik und Therapie derartiger Verletzungen vertraut sein sollten.
Mit Sprengstoffanschlägen wie in Madrid, London und kürzlich in Moskau, bei denen 280 Menschen starben und über 2000 Menschen verletzt wurden, muss letztendlich auch in Deutschland gerechnet werden. Um eine suffiziente Behandlung von Anschlagsopfern gewährleisten zu können, sind spezielle Kenntnisse unabdingbar. Dies trifft in besonderem Maße für schwere Verletzungen im Kopfund Halsbereich zu, die meist lebensbedrohlich sind und ohne eine rasche zielgerichtete Therapie häufig fatal verlaufen.

Daher spielt die Akutversorgung bei derartigen Verletzungsmustern, die in der Arbeit von Helm et al. in diesem Heft dargestellt wird, für die betroffenen Patienten eine entscheidende Rolle. Grundlage der Erstversorgung ist wie bei jedem Polytrauma der A-B-C-D-E-Algorithmus, wobei neben der Sicherung des Atemwegs v. a. die Blutstillung und Kreislaufstabilisierung im Vordergrund stehen. Die Intubation gilt nach wie vor als Goldstandard. Bei Schuss- bzw. Splitterverletzungen im Gesichts- und Halsbereich, mit starker Blutung und unübersichtlichen Verhältnissen sollte jedoch nicht zu viel Zeit mit frustranen Intubationsversuchen verloren gehen und eine chirurgische Atemwegssicherung, bevorzugt eine Koniotomie, durchgeführt werden. Für die Akutversorgung von Blutungen im Bereich des oberen Aerodigestivtrakts stehen Ballonkatheter, stark saugfähiges Verbandsmaterial und moderne Hämostyptika zur Verfügung.
Schuss- und Splitterverletzungen sind grundsätzlich als bakteriell kontaminiert zu betrachten. Daher sollte so früh wie möglich, d. h. im Rahmen der Akutversorgung eine Antibiotikaprophylaxe durchgeführt werden. Nach derzeitigen Empfehlungen eignet sich die Gabe von Cefazolin, Ceftriaxone oder Amoxicillin + Clavulansäure über 24 h gut für diesen Zweck. Bei Penicillinallergikern kann Clindamycin eingesetzt werden.

\section{$($ Die Akutversorgung spielt bei Schuss- und Splitterverletzun- gen eine entscheidende Rolle}

Jedoch ist nicht nur die lebensrettende Akutversorgung, sondern auch die definitive Versorgung, die sowohl eine zufriedenstellende funktionelle Wiederherstellung als auch ein akzeptables ästhetisches Ergebnis gewährleisten soll, außerordentlich anspruchsvoll. Im Kopf- und Halsbereich sind zahlreiche funktionell wichtige Strukturen auf engem Raum lokalisiert. Entsprechend muss mit synchronen Verletzungen von Schädelbasis und Gesichtsschädel, Augen, Gesichtsweichteilen, Nase, Ohren und/oder Organstrukturen des oberen Atmungs- und Verdauungstrakts gerechnet werden, die eine fachübergreifende Behandlung, wie sie in modernen Kopfkliniken gegeben ist, erforderlich machen. Die interdisziplinäre Zusammenarbeit zwischen erfahrenen Neurochirurgen, interventionellen Neuroradiologen, MKG-Chirurgen, 
Augenärzten, Gefäß- und HNO-Chirurgen sowie der Einsatz moderner apparativer Verfahren, wie z. B. der Computernavigation, ermöglichen eine optimale Versorgung komplexer ballistischer Verletzungen. Gleichwohl muss man bei realistischer Betrachtung feststellen, dass bei einem Massenanfall von Verletzten z. B. im Gefolge eines terroristischen Anschlags, derartige Idealbedingungen wahrscheinlich nicht allen Betroffenen zur Verfügung stehen können.

Der Zeitpunkt der definitiven chirurgischen Versorgung nach ballistischen Traumen wird kontrovers diskutiert und muss im Einzelfall individuell festgelegt werden. Dies gilt nach wie vor, wenn die Voraussetzungen hierfür gegeben sind. $\mathrm{Zu}$ fordern ist, dass der Patient stabilisiert wird und eine ausreichende klinische Infrastruktur und chirurgische Kompetenz vor Ort verfügbar sind. Wenn dies nicht der Fall ist, z. B. bei einem Massenanfall von Schwerverletzten in einer Großstadt oder bei einem Kampfeinsatz in entlegenen Regionen, ist einer verzögerten definitiven Versorgung Vorzug zu geben. Mittlerweile geht man davon aus, dass bei sachgerechter Erstversorgung für den $\mathrm{Pa}$ tienten dabei kein relevanter Nachteil entsteht.

Eine wichtige Voraussetzung für eine adäquate chirurgische Versorgung von Schuss- und Splitterverletzungen im Kopf- und Halsbereich sind wundballistische Grundkenntnisse, wie sie in der Arbeit von Hauer et al. in diesem Heft dargestellt werden. Die Schwere der Verletzung ist neben der Energie und der Oberfläche des Geschosses von der Beschaffenheit des verletzten Gewebes abhängig. Besonders tiefgreifende Verletzungen werden durch Hochrasanzgeschosse und durch Sprengsätze verursacht.

\section{() Wundballistische Grundkenntnisse sind bei der Versorgung äußerst wichtig}

Die Behandlung von Schuss- und Explosionsverletzungen im Kopf- und Halsbereich erfolgt in Abhängigkeit von der Schwere der Verletzung und den betroffenen Organstrukturen. Im vorliegenden Heft wird dabei v. a. auf Verletzun- gen der Schädelbasis, des Gesichtsschädels, der Augen und Lider, des Ohres, der Speicheldrüsen und des oberen Atmungsund Verdauungstrakts eingegangen.

Als erster Schritt muss eine Reinigung der Wunde mit vorsichtigem Débridement erfolgen. Wenn möglich erfolgt dann ein primärer spannungsfreier Verschluss von Haut- und Schleimhautläsionen. Hierbei muss allerdings darauf geachtet werden, dass durch diese Maßnahmen keine Nachteile für die definitive Rekonstruktion resultieren. Eine insuffiziente Primärversorgung kann sowohl zu funktionellen als auch zu ästhetischen, sekundär nur schwer zu korrigierenden Defiziten führen. Wenn der erstbehandelnde Arzt keine ausreichende traumatologische Erfahrung besitzt, insbesondere hinsichtlich der Behandlung von ballistischen Traumen, sollte besser eine zeitverzögerte Versorgung in einem spezialisierten Zentrum erfolgen.

Die ästhetische und funktionelle Rehabilitation stellt den dritten und abschließenden Schritt dar. Jeder einzelne Therapieabschnitt kann dabei mehrere operative Eingriffe beinhalten. Bei komplexen Traumen spielen neben manuellem Geschick und Erfahrung des behandelnden Kopf- und Halschirurgen moderne technische Hilfsmittel, wie z. B. die Anwendung der computerassistierten Chirurgie bei der Versorgung von Verletzungen des Gesichtsschädels, eine zunehmende Rolle. Die Arbeit von Schramm und Wilde in diesem Heft verdeutlicht, dass mit diesem Verfahren u. a. intraoperative Fehlpositionierungen und Fehlkonturierungen von knöchernen Fragmenten und eingebrachten Transplantaten verhindert werden können. Damit wird ein herausragender Beitrag zur Qualitätssicherung geleistet und das Risiko notwendiger Folgeeingriffe bei diesen nicht nur körperlich, sondern meist auch psychisch traumatisierten Patienten verringert.

Der Diagnostik und Therapie penetrierender Verletzungen und insbesondere ballistischer Traumen im Kopf- und Halsbereich wurde in Deutschland bislang wenig Aufmerksamkeit gewidmet. In einer Zeit, in der derartige Verletzungen nicht mehr auf Kriegsschauplätze begrenzt sind, sondern z. B. durch den inter- nationalen Terrorismus die Bevölkerung in europäischen Städten bedrohen, sind Grundkenntnisse in diesem Bereich für jeden Arzt von Bedeutung.

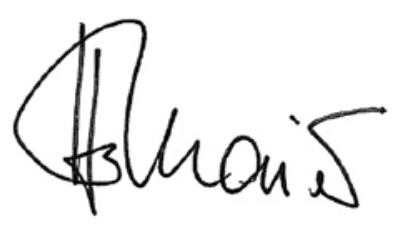

Prof. Dr. H. Maier

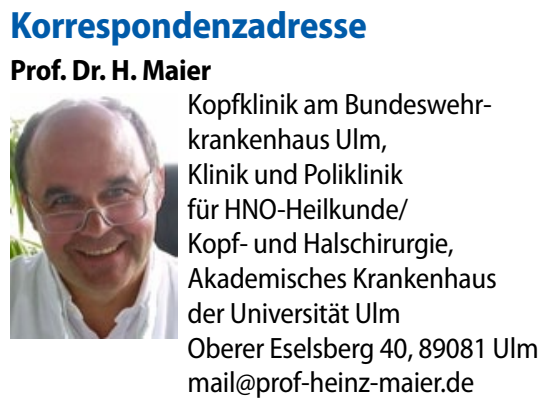

